Bull. Korean Math. Soc. 50 (2013), No. 1, pp. 105-116

http://dx.doi.org/10.4134/BKMS.2013.50.1.105

\title{
CRITICAL FUJITA EXPONENT FOR A FAST DIFFUSIVE EQUATION WITH VARIABLE COEFFICIENTS
}

\author{
Zhongping Li, Chunlai Mu, and Wanjuan Du
}

\begin{abstract}
In this paper, we consider the positive solution to a Cauchy problem in $\mathbb{R}^{N}$ of the fast diffusive equation: $|x|^{m} u_{t}=\operatorname{div}\left(|\nabla u|^{p-2} \nabla u\right)+$ $|x|^{n} u^{q}$, with nontrivial, nonnegative initial data. Here $\frac{2 N+m}{N+m+1}<p<$ $2, q>1$ and $0<m \leq n<q m+N(q-1)$. We prove that $q_{c}=p-1+\frac{p+n}{N+m}$ is the critical Fujita exponent. That is, if $1<q \leq q_{c}$, then every positive solution blows up in finite time, but for $q>q_{c}$, there exist both global and non-global solutions to the problem.
\end{abstract}

\section{Introduction}

In 1966, Fujita [4] considered the following initial value problem

$$
\begin{array}{ll}
u_{t}=\triangle u+u^{p}, & x \in \mathbb{R}^{N}, t>0, \\
u(x, 0)=u_{0}(x), & x \in \mathbb{R}^{N},
\end{array}
$$

where $N \geq 1, p>1$ and $u_{0}(x)$ is a bounded positive continuous function. They proved that the problem (1.1) does not have any nontrivial, nonnegative global solution if $1<p<p_{c}=1+\frac{2}{N}$, whereas if $p>p_{c}$, there exist both global and blowing up solutions. Such a number $p_{c}$ is then called to be the critical Fujita exponent. In [10, 29], Hayakawa and Weissler have shown that $p_{c}=1+\frac{2}{N}$ belongs to the blow-up case. These elegant works revealed a new phenomenon of nonlinear PDEs and stimulated the study of similar features for various nonlinear evolution equations (see, e.g. the survey papers [1, 12] and the references therein, and also the recent papers [4-11, 13-24, 26-29, 32]).

The critical Fujita exponent for the following Cauchy problem was given as $p_{c}=m+\frac{2}{N}$.

$$
\begin{array}{ll}
u_{t}=\triangle u^{m}+u^{p}, & x \in \mathbb{R}^{N}, t>0, \\
u(x, 0)=u_{0}(x), & x \in \mathbb{R}^{N},
\end{array}
$$

Received May 12, 2011; Revised October 19, 2011.

2010 Mathematics Subject Classification. 35K50, 35K55, 35B33.

Key words and phrases. critical Fujita exponent, fast diffusive equation, variable coefficients. 
where $p>1, m>1$ or $1>m>\frac{(N-2)_{+}}{N}$ and $u_{0}(x)$ is a bounded positive continuous function. In $[7,8,23]$ they have proved that the solution $u(x, t)$ of (1.2) blows up if $1<p<p_{c}$; while both global and nonglobal positive solutions exist if $p>p_{c}$. When $p_{c}=m+\frac{2}{N}$, in $[18,19]$ Mochizuki, Mukai and Suzuki have proved that the solutions of (1.2) blow up in finite time (see also $[6,9]$ ).

Qi [21] replaced the constant coefficient of the nonlinear source in (1.2) by the positive function $|x|^{\sigma}$ to get the equation

$$
\begin{array}{ll}
u_{t}=\triangle u^{m}+|x|^{\sigma} u^{p}, & x \in \mathbb{R}^{N}, t>0, \\
u(x, 0)=u_{0}(x), & x \in \mathbb{R}^{N},
\end{array}
$$

and established the critical Fujita exponent $p_{c}=m+\frac{2+\sigma}{N}$ for $m>\frac{(N-2)_{+}}{N}$.

In [26], some Fujita type results for (1.1), (1.2) and (1.3) were extended to

$$
|x|^{m} u_{t}=\triangle u^{k}+|x|^{n} u^{q}, \quad x \in \mathbb{R}^{N}, t>0,
$$

with $q>k \geq 1$ and $0<m \leq n<q m+q-1$ and the critical Fujita exponent of (1.4) was given as $q_{c}=k+\frac{2+n}{N+m}$.

The Cauchy problem of another nonlinear diffusive equation of the form

$$
u_{t}=\operatorname{div}\left(|\nabla u|^{p-2} \nabla u\right)+|x|^{n} u^{q}, \quad p>\frac{N+1}{2 N}, q>1,
$$

was also considered by some authors. For the problem (1.5) with $p>2$ and $n=0$, Galaktionov and Qi $[5,6,20,22]$ obtained that $q_{c}=p-1+\frac{p}{N}$ is the critical Fujita exponent of (1.5) and $q_{c}$ belongs to the blow-up case. If $n \neq 0$ in (1.5), Qi and Wang [24] proved that the critical Fujita exponent $q_{c}=p-1+\frac{p+n}{N}$ for $\frac{N+1}{2 N}<p<2$.

Recently, Martynenko and Tedeev [16, 17] studied the Cauchy problems of the following two equations with variable coefficients:

$$
\rho(x) u_{t}=\operatorname{div}\left(u^{m-1}|\nabla u|^{\lambda-1} \nabla u\right)+u^{p}, \quad x \in \mathbb{R}^{N}, t>0
$$

and

$$
\rho(x) u_{t}=\operatorname{div}\left(u^{m-1}|\nabla u|^{\lambda-1} \nabla u\right)+\rho(x) u^{p}, \quad x \in \mathbb{R}^{N}, t>0,
$$

where $\lambda>0, m+\lambda-2>0, p>m+\lambda-1, \rho(x)=|x|^{-n}$ or $\rho(x)=(1+|x|)^{-n}$. It was shown that under some restrictions on the parameters, any nontrivial solution to the Cauchy problem blows up in a finite time. Moreover, the authors established a sharp universal estimate of the solution near the blow-up point.

In this paper, we consider the positive solution to the Cauchy problem of a fast diffusive equation with variable coefficients

$$
\begin{aligned}
& |x|^{m} u_{t}=\operatorname{div}\left(|\nabla u|^{p-2} \nabla u\right)+|x|^{n} u^{q}, \quad x \in \mathbb{R}^{N}, t>0, \\
& u(x, 0)=u_{0}(x), \quad x \in \mathbb{R}^{N},
\end{aligned}
$$

where $\frac{2 N+m}{N+m+1}<p<2, q>1,0<m \leq n<q m+N(q-1)$ and $u_{0}(x)$ is a nontrivial, nonnegative, bounded and appropriately smooth function. The equation in (1.6) appears in different models in non-Newtonian fluids, here 
(1.6) may be used to describe the temperature $u$ of the channel flow of a fluid; the term $|x|^{m}$ corresponds to the reciprocal of the diffusivity; thus due to $m>0$, it has the effect of raising the temperature for $|x|<1$ and decreasing the temperature for $|x|>1$ (see [25,31], where a more detailed physical background can be found). Since $m>0$ and $\frac{2 N+m}{N+m+1}<p<2$, the equation in (1.6) is fast diffusive, which has the infinite speed of propagation property, and hence the solutions of (1.6) become instantaneously positive everywhere. That is the reason why we are restricting ourselves to positive solutions in this paper.

In this work, we are interested in the large time behavior of solutions of the Cauchy problem (1.6) and establish the critical Fujita exponent $q_{c}=p-$ $1+\frac{p+n}{N+m}$. Namely, we will prove that the solutions of the problem (1.6) have the following properties: (i) If $1<q<q_{c}$, then each positive solution blows up in a finite time. (ii) If $q>q_{c}$, then there exist both nontrivial global and nonglobal solutions with small and large $u_{0}$, respectively. Unfortunately, we assume $p>\frac{2 N}{N+1}$ instead of $p>\frac{2 N+m}{N+m+1}$ when we prove that $q_{c}=p-1+\frac{p+n}{N+m}$ belongs to blow-up case. In this case we are not able to close the gap appearing for $N>1$ between $p=\frac{2 N+m}{N+m+1}$ and $p=\frac{2 N}{N+1}$ since $\frac{2 N+m}{N+m+1}<\frac{2 N}{N+1}$ when $N>1$; we believe, however, that this is mainly due to technical difficulties, and that for $N>1$ the behavior is actually the same as for $N=1$.

We say that $u(x, t)$ is the solution to the problem (1.6) in $Q_{T}=\mathbb{R}^{N} \times(0, T)$ if $u(x, t) \in C\left(Q_{T}\right), D u \in L_{1 o c}^{1}\left(0, T ; L_{1 o c}^{1}\left(\mathbb{R}^{N}\right)\right)$ and $(1.6)$ is satisfied in the sense of distribution in $Q_{T}$, where $T>0$ is the maximal existence time. The local existence in time and uniqueness of solutions and the comparison principle for the problem (1.6) can be found in $[2,30]$. In this context, the solution $u(x, t)$ is called blow-up in finite time $T>0$ if $w(t)=\int_{\Omega} u(x, t) d x \rightarrow+\infty$ as $t \rightarrow T^{-}$ for a finite $T>0$, where $\Omega$ is a bounded domain in $\mathbb{R}^{N}$. It follows easily that this definition is the same as that of Friedman and McLeod [3] since $u(x, t)$ is a continuous function in $Q_{T}$.

When we consider the blow-up case, without loss of generality, we assume that $u_{0}(x)$ is radially symmetric and nonincreasing, i.e., $u_{0}(x)=u(r)$ with $r=|x|$, and $u_{0}(r)$ is nonincreasing in $r$. Therefore, by the comparison principle, we have that the solution of (1.6) is also radially symmetric and non-increasing in $r=|x|$. Since the solutions of (1.6) become instantaneously positive everywhere, we can assume that initial data $u_{0}(x)$ is a positive function. If $u_{0}(x)$ is not radially symmetric and non-increasing, we consider the (nonincreasing) solution $v$ to (1.6) corresponding to the initial value $v_{0}(x)=\inf \left\{u_{0}(y), 0 \leq\right.$ $|y| \leq|x|\}$, which is radially symmetric and nonincreasing in $r$. If $v$ blows up in finite time, so does $u$.

This paper is organized as follows. In Section 2 we establish the critical Fujita exponent $q_{c}$ and the critical case $q=q_{c}$ is investigated subsequently in Section 3. 


\section{Critical Fujita exponent}

In this section we give the critical exponent of Fujita type. That is, we shall show when all solutions of (1.6) blow up in a finite time or both global and nonglobal solutions exist.

Theorem 2.1. If $1<q<p-1+\frac{p+n}{N+m}$, then every nontrivial nonnegative solution of the problem (1.6) blows up in finite time.

Proof. Let

$$
\phi(x)=\left\{\begin{aligned}
1, & 0 \leq|x| \leq 1 \\
\cos \frac{1}{2}(|x|-1) \pi, & 1<|x|<2, \\
0, & |x| \geq 2
\end{aligned}\right.
$$

It follows that for $l>0, \phi_{l}(x)=\phi\left(\frac{x}{l}\right)$ is a smooth, radially symmetric, and non-increasing function which satisfies

$$
\left|\nabla \phi_{l}\right| \leq \frac{C}{l}, \quad\left|\triangle \phi_{l}\right| \leq \frac{C}{l^{2}}, \quad \frac{\left|\triangle \phi_{l}\right|}{\phi_{l}} \leq \frac{C}{l^{2}} \text { for } l<|x|<2 l,
$$

where and in the sequel $C$ is used to represent positive constant independent of $l$, and may change from line to line. Multiplying the equation in (1.6) by $\phi_{l}$ and integrating it over $\mathbb{R}^{N}$, we obtain

$$
\begin{aligned}
\frac{d}{d t} \int_{\mathbb{R}^{N}}|x|^{m} u \phi_{l} d x & =\int_{\mathbb{R}^{N}} \operatorname{div}\left(|\nabla u|^{p-2} \nabla u\right) \phi_{l} d x+\int_{\mathbb{R}^{N}}|x|^{n} u^{q} \phi_{l} d x \\
& \geq-a(n) \int_{l}^{2 l}\left|u^{\prime}\right|^{p-1}\left|\phi_{l}^{\prime}\right| r^{N-1} d r+\int_{\mathbb{R}^{N}}|x|^{n} u^{q} \phi_{l} d x,
\end{aligned}
$$

here $a(n)$ denotes the volume of the unit ball in $\mathbb{R}^{N}$. Using Hölder's inequality, we have

$$
\begin{aligned}
& \int_{l}^{2 l}\left|u^{\prime}\right|^{p-1}\left|\phi_{l}^{\prime}\right| r^{N-1} d r \\
\leq & \left(\int_{l}^{2 l}\left|u^{\prime}\right|\left|\phi_{l}^{\prime}\right| r^{N-1} d r\right)^{p-1}\left(\int_{l}^{2 l}\left|\phi_{l}^{\prime}\right| r^{N-1} d r\right)^{2-p} .
\end{aligned}
$$

Note that $\frac{\partial \phi_{l}}{\partial \nu}=0$ on $\partial B_{l}$ and $\frac{\partial \phi_{l}}{\partial \nu} \leq 0$ on $\partial B_{2 l}$, where $B_{l}$ is the ball in $\mathbb{R}^{N}$ with radius $l$ and center at the origin, and $\nu$ is the outward normal vector on the boundary $\partial B_{l}$ or $\partial B_{2 l}$. Since $u$ and $\phi_{l}$ are radially symmetric, and non-increasing functions, our employing polar coordinates gives

$$
\begin{aligned}
\int_{l}^{2 l}\left|u^{\prime}\right|\left|\phi_{l}^{\prime}\right| r^{N-1} d r & =\frac{1}{a(n)} \int_{B_{2 l} \backslash B_{l}} \nabla u \cdot \nabla \phi_{l} d x \\
& \leq \frac{1}{a(n)}\left|\int_{\partial B_{2 l}} u \frac{\partial \phi_{l}}{\partial \nu} d S-\int_{B_{2 l} \backslash B_{l}} \nabla u \cdot \nabla \phi_{l} d x\right| \\
& =\frac{1}{a(n)}\left|\int_{\partial\left(B_{2 l} \backslash B_{l}\right)} u \frac{\partial \phi_{l}}{\partial \nu} d S-\int_{B_{2 l} \backslash B_{l}} \nabla u \cdot \nabla \phi_{l} d x\right|
\end{aligned}
$$




$$
\begin{aligned}
& =\frac{1}{a(n)}\left|\int_{B_{2 l} \backslash B_{l}} u \Delta \phi_{l} d x\right| \\
& \leq \frac{1}{a(n)} \int_{B_{2 l} \backslash B_{l}} u\left|\triangle \phi_{l}\right| d x .
\end{aligned}
$$

We continue to by using Hölder's inequality to discover

$$
\begin{aligned}
& \int_{B_{2 l} \backslash B_{l}} u\left|\triangle \phi_{l}\right| d x \\
\leq & \left(\int_{B_{2 l} \backslash B_{l}}|x|^{n} u^{q} \phi_{l} d x\right)^{\frac{1}{q}}\left(\int_{B_{2 l} \backslash B_{l}}\left(|x|^{-n}\left|\triangle \phi_{l}\right|^{q} \phi_{l}^{-1}\right)^{\frac{1}{q-1}} d x\right)^{\frac{q-1}{q}} .
\end{aligned}
$$

Next by a direct computation we have

$$
\begin{array}{r}
\left(\int_{l}^{2 l}\left|\phi_{l}^{\prime}\right| r^{N-1} d r\right)^{2-p}=C l^{(N-1)(2-p)}, \\
\left(\int_{B_{2 l} \backslash B_{l}}\left(|x|^{-n}\left|\triangle \phi_{l}\right|^{q} \phi_{l}^{-1}\right)^{\frac{1}{q-1}} d x\right)^{\frac{q-1}{q}}=C l^{\frac{N(q-1)-n-2 q}{q} .}
\end{array}
$$

Combining (2.2)-(2.5), we find

$$
\begin{aligned}
& \int_{l}^{2 l}\left|u^{\prime}\right|^{p-1}\left|\phi_{l}^{\prime}\right| r^{N-1} d r \\
\leq & C l^{(N-1)(2-p)+\frac{[N(q-1)-n-2 q](p-1)}{q}}\left(\int_{\mathbb{R}^{N}}|x|^{n} u^{q} \phi_{l} d x\right)^{\frac{p-1}{q}} .
\end{aligned}
$$

Set

$$
w_{l}(t)=\int_{\mathbb{R}^{N}}|x|^{m} u \phi_{l} d x, t>0 .
$$

Substituting (2.6) into (2.1) yields

$$
\begin{array}{r}
\frac{d w_{l}}{d t}=\left(\int_{\mathbb{R}^{N}}|x|^{n} u^{q} \phi_{l} d x\right)^{\frac{p-1}{q}}\left(-C l^{(N-1)(2-p)+\frac{[N(q-1)-n-2 q](p-1)}{q}}\right. \\
\left.+\left(\int_{\mathbb{R}^{N}}|x|^{n} u^{q} \phi_{l} d x\right)^{\frac{q-p+1}{q}}\right) .
\end{array}
$$

By the Hölder's inequality with $q m-n>-N(q-1)$, we have

$$
\begin{aligned}
\int_{\mathbb{R}^{N}}|x|^{m} u \phi_{l} d x & \leq\left(\int_{\mathbb{R}^{N}}|x|^{\frac{q m-n}{q-1}} \phi_{l} d x\right)^{\frac{q-1}{q}}\left(\int_{\mathbb{R}^{N}}|x|^{n} u^{q} \phi_{l} d x\right)^{\frac{1}{q}} \\
& \leq C l^{N+m-\frac{N+n}{q}}\left(\int_{\mathbb{R}^{N}}|x|^{n} u^{q} \phi_{l} d x\right)^{\frac{1}{q}}
\end{aligned}
$$


i.e.,

$$
\int_{\mathbb{R}^{N}}|x|^{n} u^{q} \phi_{l} d x \geq C w_{l}^{q} l^{-q N-q m+N+n} .
$$

According to (2.7) and (2.8), we obtain

$$
\begin{aligned}
\frac{d w_{l}}{d t} \geq l^{\frac{(-q N-q m+N+n)(p-1)}{q}} w_{l}^{p-1}( & -C_{1} l^{(N-1)(2-p)+\frac{[N(q-1)-n-2 q](p-1)}{q}} \\
& \left.+C_{2} w_{l}^{q-p+1} l^{\frac{(-q N-q m+N+n)(q-p+1)}{q}}\right) .
\end{aligned}
$$

Under the assumption $q<p-1+\frac{p+n}{N+m}$, we see

$(N-1)(2-p)+\frac{[N(q-1)-n-2 q](p-1)}{q}<\frac{(-q N-q m+N+n)(q-p+1)}{q}$.

Thus using the fact that $w_{l}$ is an increasing function of $l$, we find from (2.9) that, for sufficiently large $l$, there exists a constant $\delta>0$ such that

$$
\frac{d w_{l}}{d t} \geq \delta w_{l}^{q}, \quad t>0 .
$$

Recalling that supp $\phi_{l}=B_{2 l}$, we then follow $w_{l}$, and consequently $u$, blows up in finite time since $q>1$.

Theorem 2.2. If $q>p-1+\frac{p+n}{N+m}$, then the solution of the problem (1.6) with appropriately large initial data blows up in finite time.

Proof. We know from the proof of Theorem 2.1 that $u$ satisfies (2.9). If $u_{0}$ is sufficiently large such that, for some $l>0$,

$$
C_{1} l^{(N-1)(2-p)+\frac{[N(q-1)-n-2 q](p-1)}{q}} \leq \frac{1}{2} C_{2} w_{l}^{q-p+1}(0) \frac{(-q N-q m+N+n)(q-p+1)}{q} .
$$

Then, from (2.9), we see that

$$
w_{l}(t) \geq w(0), \quad t>0
$$

and

$\frac{d w_{l}}{d t} \geq l^{\frac{(-q N-q m+N+n)(p-1)}{q}} w_{l}^{p-1}\left(\frac{1}{2} C_{2} w_{l}^{q-p+1} l^{\frac{(-q N-q m+N+n)(q-p+1)}{q}}\right) \geq \delta w_{l}^{q}, t>0$, for some $\delta>0$. This verifies that $u$ blows up in finite time.

Theorem 2.3. If $q>p-1+\frac{p+n}{N+m}$, then the problem (1.6) admits global solutions with small initial data.

Proof. We investigate the auxiliary function

$$
\bar{u}(x, t)=(t+1)^{-\alpha} f(\xi), \quad \xi=|x|(t+1)^{-\beta},
$$

where $f(\xi)$ is to be determined later and

$$
\alpha=\frac{p+n}{m(q-p+1)+n(p-2)+p(q-1)}, \quad \beta=\frac{q-p+1}{p+n} \alpha .
$$


Then we have

$$
\begin{aligned}
\bar{u}_{t} & =(t+1)^{-(\alpha+1)}\left(-\alpha f(\xi)-\beta \xi f^{\prime}(\xi)\right), \\
|\nabla \bar{u}|^{p-2} \nabla \bar{u} & =(t+\tau)^{-(p-1)(\alpha+\beta)}\left|f^{\prime}\right|^{p-2} f^{\prime}(\xi) \nabla|x|, \\
\operatorname{div}\left(\left.\nabla \bar{u}\right|^{p-2} \nabla \bar{u}\right) & =(t+1)^{-(p-1) \alpha-p \beta}\left(\left(\left|f^{\prime}\right|^{p-2} f^{\prime}\right)^{\prime}(\xi)+\frac{N-1}{\xi}\left|f^{\prime}\right|^{p-2} f^{\prime}(\xi)\right) .
\end{aligned}
$$

$\bar{u}(x, t)$ is a supersolution of the problem (1.6) with small initial data $u_{0}$ if the function $f(\xi)$ satisfies

$$
\begin{aligned}
& \left(\left|f^{\prime}\right|^{p-2} f^{\prime}\right)^{\prime}(\xi)+\frac{N-1}{\xi}\left|f^{\prime}\right|^{p-2} f^{\prime}(\xi)+\beta \xi^{m+1} f^{\prime}(\xi)+\alpha \xi^{m} f(\xi)+\xi^{n} f^{q}(\xi) \leq 0, \\
& \xi>0, \quad f(0)>0, \quad f^{\prime}(0)=0 .
\end{aligned}
$$

Take

$$
f(\xi)=\left(a+A \xi^{\frac{p+m}{p-1}}\right)^{-\frac{p-1}{2-p}},
$$

where $a, A>0$ are constants to be chosen.

After a computation, we have

$$
\begin{aligned}
f^{\prime}(\xi)= & -\frac{p+m}{2-p} A\left(a+A \xi^{\frac{p+m}{p-1}}\right)^{-\frac{1}{2-p}} \xi^{\frac{m+1}{p-1}} \\
\mid\left(\left.f^{\prime}\right|^{p-2} f^{\prime}(\xi)=\right. & -\left(\frac{p+m}{2-p}\right)^{p-1} A^{p-1}\left(a+A \xi^{\frac{p+m}{p-1}}\right)^{-\frac{p-1}{2-p}} \xi^{m+1} \\
\left(\left|f^{\prime}\right|^{p-2} f^{\prime}\right)^{\prime}(\xi)= & \left(\frac{p+m}{2-p}\right)^{p} A^{p}\left(a+A \xi^{\frac{p+m}{p-1}}\right)^{-\frac{1}{2-p}} \xi^{\frac{p(m+1)}{p-1}} \\
& -(m+1)\left(\frac{p+m}{2-p}\right)^{p-1} A^{p-1}\left(a+A \xi^{\frac{p+m}{p-1}}\right)^{-\frac{p-1}{2-p}} \xi^{m} .
\end{aligned}
$$

Then inserting the expression of $f(\xi)$ into the first inequality in $(2.11)$, we obtain

$$
\begin{aligned}
& \frac{p+m}{2-p} A\left(a+A \xi^{\frac{p+m}{p-1}}\right)^{-\frac{1}{2-p}} \xi^{\frac{p(m+1)}{p-1}}\left[\left(\frac{p+m}{2-p}\right)^{p-1} A^{p-1}-\beta\right] \\
& +\left(a+A \xi^{\frac{p+m}{p-1}}\right)^{-\frac{p-1}{2-p}} \xi^{m}\left[\alpha-(N+m)\left(\frac{p+m}{2-p}\right)^{p-1} A^{p-1}\right] \\
& +\xi^{n}\left(a+A \xi^{\frac{p+m}{p-1}}\right)^{-\frac{(p-1) q}{2-p}} \leq 0
\end{aligned}
$$

namely,

$$
\begin{aligned}
& \frac{p+m}{2-p} A\left(a+A \xi^{\frac{p+m}{p-1}}\right)^{\frac{p-2}{2-p}} \xi^{\frac{p+m}{p-1}}\left[\left(\frac{p+m}{2-p}\right)^{p-1} A^{p-1}-\beta\right] \\
& +\left[\alpha-(N+m)\left(\frac{p+m}{2-p}\right)^{p-1} A^{p-1}\right] \\
& +\xi^{n-m}\left(a+A \xi^{\frac{p+m}{p-1}}\right)^{-\frac{(p-1)(q-1)}{2-p}} \leq 0 .
\end{aligned}
$$


Noting that $q>p-1+\frac{p+n}{N+m}$ implies that $\beta>\frac{\alpha}{N+m}$, we choose $A$ such that

and define

$$
\frac{\alpha}{N+m}\left(\frac{2-p}{p+m}\right)^{p-1}<A^{p-1}<\beta\left(\frac{2-p}{p+m}\right)^{p-1}
$$

By taking

$$
\sigma=(N+m)\left(\frac{p+m}{2-p}\right)^{p-1} A^{p-1}-\alpha
$$

$$
a \geq A^{-\frac{(n-m)(2-p)}{(p+m)(q-1)-(n-m)(2-p)}} \sigma^{-\frac{(p+m)(2-p)}{[(p+m)(q-1)-(n-m)(2-p)](p-1)}},
$$

for every $\xi>0$, we have

$$
\xi^{n-m}\left(a+A \xi^{\frac{p+m}{p-1}}\right)^{-\frac{(p-1)(q-1)}{2-p}} \leq \sigma .
$$

Thus, with this choice of $a, A$, from (2.13) we check that the first inequality of (2.11) is valid. Thus, for the case $q>q_{c}$, we have constructed a class of global self-similar supersolutions defined by (2.10) and (2.12). Owing to the comparison principle, the solution of the problem (1.6) is global if the initial data $u_{0}$ is small enough.

\section{The critical case $q=q_{c}$}

In order to study the critical case $q=q_{c}$, we give a large time behavior of the solution to (1.6) for $|x|>1$.

Lemma 3.1. The positive solution of the problem (1.6) has, for each $t \in(\tau, T)$,

$$
u(x, t) \geq \epsilon(t-\tau)^{-\alpha}\left(1+\delta r^{k}\right)^{-\gamma}, \quad|x|>1,
$$

where $T$ is the maximal existence time for the solution, $\epsilon, \delta$ are positive constants and

$$
\begin{aligned}
\alpha=\frac{N+m}{(p-1)(N+m)+p-N}, & \beta=\frac{1}{(p-1)(N+m)+p-N}, \\
k=\frac{p+m}{p-1}, \quad \gamma=\frac{p-1}{2-p}, & r=|x|(t-\tau)^{-\beta} .
\end{aligned}
$$

Proof. Our idea is to show that any positive solution of the problem (1.6) is, for $|x|>1$, bigger than the following similarity solution

$$
U_{\lambda}(t, x)=\lambda^{\frac{p+m}{2-p}} U(t, \lambda x),
$$

where

$$
U(t, x)=t^{-\frac{N+m}{(p-1)(N+m)+p-N}}\left(1+b|x|^{\frac{p+m}{p-1}} t^{-\frac{p+m}{[(p-1)(N+m)+p-N](p-1)}}\right)^{-\frac{p-1}{2-p}}
$$

with $b=\frac{2-p}{p+m} \beta^{\frac{1}{p-1}}$. Let $0<\tau<T_{*}<T$ and $S=\left[\tau, T_{*}\right] \times(1,+\infty)$. Since the positive solution $u(x, t)$ is continuous in $\left(0, T_{*}\right] \times[0,+\infty)$, there exists $\delta=$ $\delta\left(\tau, T_{*}\right)>0$ such that

$$
\delta=\min u(x, t), \quad \tau \leq t \leq T_{*}, 0 \leq|x| \leq 1 .
$$


We now select $\lambda>0$ such that

$$
U_{\lambda}(t-\tau, x) \leq \delta, \quad \tau \leq t \leq T_{*},|x| \geq \frac{1}{2}
$$

To this aim, according to the definition of $U_{\lambda}(t, x)$ we need

$$
\lambda^{\frac{p+m}{2-p}}(t-\tau)^{-\frac{N+m}{(p-1)(N+m)+p-N}}\left(1+b \lambda^{\frac{p+m}{p-1}}|x|^{\frac{p+m}{p-1}}(t-\tau)^{-\frac{p+m}{[(p-1)(N+m)+p-N](p-1)}}\right)^{-\frac{p-1}{2-p}} \leq \delta,
$$

or

$\delta^{\frac{p-2}{p-1}} \leq \lambda^{-\frac{p+m}{p-1}}(t-\tau)^{\frac{(N+m)(2-p)}{[(p-1)(N+m)+p-N](p-1)}}+b|x|^{\frac{p+m}{p-1}}(t-\tau)^{-\frac{p+m-(N+m)(2-p)}{[(p-1)(N+m)+p-N](p-1)}}$ for $\tau \leq t \leq T_{*}$ and $|x| \geq \frac{1}{2}$, which is implied by

$\delta^{\frac{p-2}{p-1}} \leq \lambda^{-\frac{p+m}{p-1}}(t-\tau)^{\frac{(N+m)(2-p)}{(p-1)(N+m)+p-N](p-1)}}+b\left(\frac{1}{2}\right)^{\frac{p+m}{p-1}}(t-\tau)^{-\frac{p+m-(N+m)(2-p)}{[(p-1)(N+m)+p-N](p-1)}}$.

Since the right-hand side of (3.5) is bounded below by $c \lambda^{-\frac{p+m-(N+m)(2-p)}{p-1}}$, where $c$ is a constant independent of $\lambda,(3.5)$ is satisfied if we choose $\lambda$ such that $\lambda \leq$ $c \delta^{\frac{2-p}{p+m-(N+m)(2-p)}}$. Since $|x|^{m} U_{\lambda t}=\operatorname{div}\left(\left|\nabla U_{\lambda}\right|^{p-2} \nabla U_{\lambda}\right)$ in $S$ and $U_{\lambda}(t-\tau, x)=0$ for $t=\tau,|x| \geq 1$, by (3.2), (3.3) and the comparison principle we have

$$
U_{\lambda}(t-\tau, x) \leq u(x, t), \quad \tau<t<T_{*},|x| \geq 1 .
$$

Hence, the estimate (3.1) holds by letting $T_{*}$ tend to $T$.

Theorem 3.2. If $q=p-1+\frac{p+n}{N+m}$ and $p>\frac{2 N}{N+1}$, then every positive solution of (1.6) blows up in finite time.

Proof. Assume by contradiction that $u(x, t)$ is a non-trivial global solution of the problem (1.6). Noticing that $q=p-1+\frac{p+n}{N+m}$ implies

$(N-1)(2-p)+\frac{[N(q-1)-n-2 q](p-1)}{q}=\frac{(-q N-q m+N+n)(q-p+1)}{q}$.

From (2.9), we find that

$$
C_{2} w_{l}^{q-p+1} \leq 2 C_{1}, \quad \forall l, t>0,
$$

where $C_{1}$ and $C_{2}$ are independent of $l$ and $t$. Otherwise, we can prove $w_{l}$, and hence $u$, blows up in finite time. Noting that

$$
\lim _{l \rightarrow+\infty} w_{l}(t)=\int_{\mathbb{R}^{N}}|x|^{m} u d x
$$

by (3.6) we obtain that

$$
\int_{\mathbb{R}^{N}}|x|^{m} u d x \leq C, \forall t>0
$$


Recalling (2.1)-(2.3) and (2.5), we deduce for $l>1$,

$$
\begin{aligned}
& \frac{d}{d t} \int_{\mathbb{R}^{N}}|x|^{m} u \phi_{l} d x \\
\geq & -C l^{(N-1)(2-p)}\left(\int_{B_{2 l} \backslash B_{l}} u\left|\Delta \phi_{l}\right| d x\right)^{p-1}+\int_{\mathbb{R}^{N}}|x|^{n} u^{q} \phi_{l} d x \\
= & -C l^{(N-1)(2-p)}\left(\int_{B_{2 l} \backslash B_{l}} u \phi_{l} \frac{\left|\triangle \phi_{l}\right|}{\phi_{l}} d x\right)^{p-1}+\int_{\mathbb{R}^{N}}|x|^{n} u^{q} \phi_{l} d x \\
\geq & -C l^{(N-1)(2-p)-2(p-1)}\left(\int_{B_{2 l} \backslash B_{l}} u \phi_{l} d x\right)^{p-1}+\int_{\mathbb{R}^{N}}|x|^{n} u^{q} \phi_{l} d x \\
\geq & -C l^{(N-1)(2-p)-2(p-1)}\left(\int_{B_{2 l} \backslash B_{l}}|x|^{m} u \phi_{l} d x\right)^{p-1}+\int_{\mathbb{R}^{N}}|x|^{n} u^{q} \phi_{l} d x \\
\geq & -C l^{(N-1)(2-p)-2(p-1)}\left(\int_{\mathbb{R}^{N}}|x|^{m} u \phi_{l} d x\right)^{p-1}+\int_{\mathbb{R}^{N}}|x|^{n} u^{q} \phi_{l} d x .
\end{aligned}
$$

Considering the assumptions $p>\frac{2 N}{N+1}$, we know $(N-1)(2-p)-2(p-1)<0$. Taking $l \rightarrow+\infty$ in (3.8), from (3.7) we obtain

$$
\frac{d}{d t} \int_{\mathbb{R}^{N}}|x|^{m} u d x \geq \frac{1}{2} \int_{\mathbb{R}^{N}}|x|^{n} u^{q} d x .
$$

Let $w(t)=\int_{\mathbb{R}^{N}}|x|^{m} u d x$, then

$$
w(t)-w(0) \geq \frac{1}{2} \int_{0}^{t} \int_{\mathbb{R}^{N}}|x|^{n} u^{q}(x, s) d x d s .
$$

Employing Lemma 3.1 we have

$$
\begin{aligned}
\int_{\mathbb{R}^{N}}|x|^{n} u^{q}(x, t) d x & \geq \epsilon^{q}(t-\tau)^{-1} \int_{|y| \geq(t-\tau)^{-\beta}}|y|^{n}\left(1+\delta|y|^{k}\right)^{-q \gamma} d y \\
& \geq c(t-\tau)^{-1}
\end{aligned}
$$

Then by (3.9) we have

$$
\lim _{t \rightarrow+\infty} w(t)=+\infty
$$

i.e.,

$$
\lim _{t \rightarrow+\infty} \int_{\mathbb{R}^{N}}|x|^{m} u d x=+\infty
$$

This is a contradiction to (3.7).

Acknowledgements. This work was partially supported by NNSF of China (11071266), partially supported by Projects Supported by Scientific Research Fund of SiChuan Provincial Education Department(09ZA119). 


\section{References}

[1] K. Deng and H. A. Levine, The role of critical exponents in blow-up theorems: the sequel, J. Math. Anal. Appl. 243 (2000), no. 1, 85-126.

[2] E. DiBenedetto, Degenerate Parabolic Equations, Springer-Verlag, New York, 1993.

[3] A. Friedman and J. B. McLeod, Blow-up of positive solutions of semilinear heat equation, Indiana Univ. Math. J. 34 (1985), no. 2, 425-447.

[4] H. Fujita, On the blowing up of solutions of the Cauchy problem for $u_{t}=\Delta u+u^{1+\alpha}$, J. Fac. Sci. Univ. Tokyo Sec. I 13 (1966), 109-124.

[5] V. A. Galaktionov, Conditions for global nonexistence and localization for a class of nonlinear parabolic equations, Comput. Math. Math. Phys. 23 (1983), 35-44.

[6] - Blow-up for quasilinear heat equations with critical Fujita's exponents, Proc. Roy. Soc. Edinburgh Sect. A 124 (1994), no. 3, 517-525.

[7] V. A. Galaktionov, S. P. Kurdyumov, A. P. Mikhailov, and A. A. Samarskii, On unbounded solutions of the Cauchy problem for the parabolic equation $u_{t}=\nabla\left(u^{\sigma} \nabla u\right)+u^{\beta}$, Soviet Phys. Dokl. 25 (1980), 458-459.

[8] _ Blowup in Quasilinear Parabolic Equations, De Gruyter Expositions in Mathematics, Springer, Berlin, 1995.

[9] V. A. Galaktionov and H. A. Levine, A general approach to critical Fujita exponents and systems, Nonlinear Anal. 34 (1998), no. 7, 1005-1027.

[10] K. Hayakawa, On nonexistence of global solutions of some semilinear parabolic differential equations, Proc. Japan Acad. 49 (1973), 503-505.

[11] Q. Huang, K. Mochizuki, and K. Mukai, Life span and asymptotic behavior for a semilinear parabolic system with slowly decaying initial values, Hokkaido Math. J. 27 (1998), no. 2, 393-407.

[12] H. A. Levine, The role of critical exponents in blow-up theorems, SIAM Rev. 32 (1990), no. 2, 262-288.

[13] Z. P. Li and C. L. Mu, Critical exponents for a fast diffusive polytropic filtration equation with nonlinear boundary flux, J. Math. Anal. Appl. 346 (2008), no. 1, 55-64.

[14] Z. P. Li, C. L. Mu, and L. Xie, Critical curves for a degenerate parabolic equation with multiple nonlinearities, J. Math. Anal. Appl. 359 (2009), no. 1, 39-47.

[15] Z. P. Li, C. L. Mu, and Z. J. Cui, Critical curves for a fast diffusive polytropic filtration system coupled via nonlinear boundary flux, Z. Angew. Math. Phys. 60 (2008), no. 2, 284-298.

[16] A. V. Martynenko and A. F. Tedeev, The Cauchy problem for a quasilinear parabolic equation with a source and nonhomogeneous density, Comput. Math. Math. Phys. 47 (2007), no. 2, 238-48.

[17] On the behavior of solutions to the Cauchy problem for a degenerate parabolic equation with inhomogeneous density and a source, Comput. Math. Math. Phys. 48 (2008), no. 7, 1145-1160.

[18] K. Mochizuki and K. Mukai, Existence and nonexistence of global solutions to fast diffusions with source, Methods Appl. Anal. 2 (1995), no. 1, 92-102.

[19] K. Mochizuki and R. Suzuki, Critical exponent and critical blow-up for quasi-linear parabolic equations, Israel J. Math. 98 (1997), no. 1, 141-156.

[20] Y. W. Qi, Critical exponents of degenerate parabolic equations, Sci. China Ser. A 38 (1995), no. 10, 1153-1162.

[21] Soc. Edinburgh Sect. A 128 (1998), no. 1, 123-136.

[22] _ The global existence and nonuniqueness of a nonlinear degenerate equation, Nonlinear Anal. 31 (1998), no. 1-2, 117-136.

[23] Y. W. Qi and H. A. Levine, The critical exponent of degenerate parabolic systems, Z. Angew. Math. Phys. 44 (1993), no. 2, 249-265. 
[24] Y. W. Qi and M. X. Wang, Critical exponents of quasilinear parabolic equations, J. Math. Anal. Appl. 267 (2002), no. 1, 264-280

[25] A. E. Scheidegger, The Physics of Flow Through Porous Media, third ed., Buffalo, Toronto, 1974

[26] C. P. Wang and S. N. Zheng, Critical Fujita exponents of degenerate and singular parabolic equations, Proc. Roy. Soc. Edinburgh Sect. A 136 (2006), no. 2, 415-430.

[27] C. P. Wang, S. N. Zheng, and Z. J. Wang, Critical Fujita exponents for a class of quasilinear equations with homogeneous Neumann boundary data, Nonlinearity 20 (2007), no. $6,1343-1359$.

[28] Z. J. Wang, J. X. Yin, C. P. Wang, and H. Gao, Large time behavior of solutions to Newtonian filtration equation with nonlinear boundary sources, J. Evol. Equ. 7 (2007), no. $4,615-648$.

[29] F. B. Weissler, Existence and nonexistence of global solutions for a semilinear heat equation, Israel J. Math. 38 (1981), no. 1-2, 29-40.

[30] Z. Q. Wu, J. N. Zhao, J. X. Yin, and H. L. Li, Nonlinear Diffusion Equations, Word Scientific, Singapore, 2001.

[31] Y. B. Zeldovich and Y. P. Raizer, Physics and shock waves and high-temperature hydrodynamic phenomena, Moscow, vol. 2, Academic Press, New York, 1967.

[32] S. N. Zheng and C. P. Wang, Large time behaviour of solutions to a class of quasilinear parabolic equations with convection terms, Nonlinearity 21 (2008), no. 9, 2179-2200.

ZHONGPING LI

College of Mathematic and Information

China West Normal University

NANChONG 637009, P. R. China

E-mail address: zhongping-li@hotmail.com

Chunlai Mu

College of mathematics and Statistics

Chongqing University

Chongqing, 401331, P. R. China

E-mail address: chunlaimu@yahoo.com.cn

WANJUAN DU

College of Mathematic and Information

China West Normal University

NANChONG 637009, P. R. China

E-mail address: duwanjuan28@163.com 